\title{
Postoperatives Management der Gerinnung
}

\author{
Julian Sassenscheidt Christian Zöllner \\ Klinik und Poliklinik für Anästhesiologie, Zentrum für Anästhesiologie und Intensivmedizin, \\ Universitätsklinikum Hamburg-Eppendorf, Hamburg, Deutschland
}

\section{Schlüsselwörter \\ Postoperative Blutung - Blutgerinnungstests · \\ Neue Antikoagulanzien · Hämostatika · Gerinnung}

\section{Zusammenfassung}

Hintergrund: Das Gerinnungsmanagement in der postoperativen Phase stellt eine komplexe Herausforderung dar. Einerseits führt eine bereits präoperativ vorbestehende oder intraoperativ erworbene Beeinträchtigung der Blutgerinnung zu einem erhöhten Risiko für Nachblutungen. Andererseits sind Patienten in der postoperativen Phase einem erhöhten Risiko für thromboembolische Komplikationen ausgesetzt. Zudem drängen vermehrt neue orale Antikoagulanzien und Thrombozytenaggregationshemmer auf den Markt, für deren perioperativen Einsatz noch wenig Erfahrung besteht. Methode: Literaturübersicht. Ergebnisse: Das postoperative Gerinnungsmanagement sollte individualisiert nach einer Diagnostik mit klassischen Gerinnungstests und Point-of-Care(POC)-Diagnoseverfahren erfolgen. Verglichen mit dem intraoperativen Gerinnungsmanagement bestehen Unterschiede. Schlussfolgerungen: Das Ziel des postoperativen Gerinnungsmanagements ist eine Gerinnungsaktivität, die eine suffiziente Wundheilung zulässt, ohne thromboembolische Ereignisse zu begünstigen. Im Falle einer postoperativen Blutung sollte zur Diagnostik neben den klassischen Gerinnungstests auf viskoelastische POC-Verfahren zurückgegriffen werden. Neben der bedarfsadaptierten Substitution von Gerinnungsfaktoren ist die Aufrechterhaltung der Rahmenbedingungen der Gerinnung von entscheidender Bedeutung (Normothermie, physiologischer pH-Wert, Normokalziämie). Die Fortführung einer bestehenden Antikoagulation mit neuen oralen Antikoagulanzien ist eine Einzelfallentscheidung. Für ihren perioperativen Einsatz gib es noch keine Leitlinien.

\section{KARGER \\ Fax +497614520714}

Information@Karger.com

www.karger.com

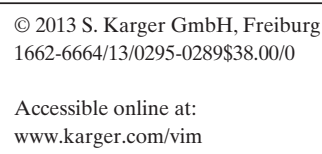

\section{Keywords}

Postoperative hemorrhage - Blood coagulation tests .

New anticoagulants · Hemostatics · Hemostasis

\section{Summary}

\section{Postoperative Coagulation Management}

Background: Postoperative coagulation management is a complex challenge. An impaired hemostasis might induce postoperative hemorrhage; however, in the early postoperative phase, patients have an elevated risk for thromboembolic events. Furthermore, there is a lack of experience for the perioperative use of the new oral anticoagulants available on the market during the last few years. Method: Literature review. Results: Postoperative coagulation management should be performed in an individualized way according to the diagnosis with classic coagulation tests and point-of-care (POC) diagnostic procedures. Compared with intraoperative coagulation management, differences can be detected. Conclusion: The aim of postoperative coagulation management is to maintain a hemostasis which is sufficient for wound healing without promoting the risk for thromboembolic events. In the case of hemorrhage, the diagnostics should be expanded to viscoelastic POC tests beside the classic coagulation tests. Maintaining temperature, $\mathrm{pH}$ value, and normocalcemia is of outstanding importance. Resumption of a therapy with the new oral anticoagulants is a case-by-case decision as guidelines are still lacking. 


\section{Einleitung}

Perioperativer Blutverlust inklusive der Transfusion von Fremdblut ist mit einer erhöhten Morbidität und Mortalität vergesellschaftet [1]. Auch wenn der Zeitpunkt der größten Dynamik perioperativer Hämorrhagien erfahrungsgemäß intraoperativ liegt, ist die postoperative Blutung neben der Sepsis und den Anastomoseninsuffizienzen ein häufiger Grund für Relaparotomien [2]. Sofern chirurgisch behebbare Ursachen ausgeschlossen sind, muss eine postoperativ neu aufgetretene oder bereits vorbestehende Koagulopathie als Auslöser der Blutung vermutet werden. Koagulopathien können angeboren (Hämophilie, von-Willebrand-Syndrom (vWS)) oder erworben (Antikoagulation, Dilutionskoagulopathie, Hyperfibrinolyse, Verbrauchskoagulopathie) sein. Im Rahmen eines operativen Eingriffs kommt es durch verschiedene Mechanismen zu einer Beeinträchtigung der Hämostase. Zum einen führt das chirurgische Trauma selbst zu einem Verbrauch von Gerinnungsfaktoren und Thrombozyten und kann durch eine Akute-Phase-Reaktion zu einem Ungleichgewicht von pro- und antikoagulatorischen Faktoren führen [3]. Zum anderen führt ein vermehrter Volumenumsatz auch in der postoperativen Phase zu einer Verdünnung von Gerinnungsfaktoren und Thrombozyten mit dem Resultat einer Dilutionskoagulopathie. Komplizierend kommt hinzu, dass der Patient in der perioperativen Phase durch Immobilisation sowie eine prokoagulatorische Wirkung der Stressantwort einem erhöhten Risiko für thromboembolische Komplikationen ausgesetzt ist, die eine medikamentöse Thromboembolieprophylaxe erfordern [4]. Des Weiteren nimmt die Anzahl an Verschreibungen von Antikoagulanzien zu [5], sodass der perioperativ betreuende Arzt immer häufiger mit Patienten unter Antikoagulation konfrontiert ist. Für den perioperativen Einsatz der sogenannten neuen Antikoagulanzien besteht zudem noch wenig Erfahrung.

\section{Koagulopathie}

\section{Pathomechanismus}

Bei operativen Eingriffen kann es in der perioperativen Phase zu vermehrten Blutverlusten kommen. Um bei einem fortgeschrittenen Blutverlust ein ausreichendes intravasales Flüssigkeitsvolumen sicherzustellen, müssen auch in der postoperativen Phase Volumenersatzmittel infundiert werden. Hierbei handelt es sich in der Regel um kristalloide oder kolloidale Flüssigkeiten sowie bei Erreichen einer individuellen kritischen Hämoglobinkonzentration um Erythrozytenkonzentrate. Durch den Austausch von Vollblut mit Volumenersatzmitteln und Erythrozytenkonzentraten kommt es zu einer Verdünnung von sämtlichen Bestandteilen der Hämostase. Das Ausmaß der Verdünnung betrifft nicht alle Faktoren und Zellen in gleichem Maße, wie es in einem In-
vitro-Modell geschehen würde [6]. Tatsächlich wird die Freisetzung von Faktor VIII und von-Willebrand-Faktor durch Stresshormone getriggert [3, 7]. Zudem wird Fibrinogen zwar als klassisches Protein der akuten Phase vermehrt sezerniert, jedoch genügt dies nicht, um den vermehrten Fibrinogenumsatz zu kompensieren. Außerdem kommt es auch zu einer Verdünnung von Antithrombin und somit zu einer verlängerten Wirkdauer von aktiviertem Faktor Xa sowie von Thrombin [8]. Weiterhin hat die Auswahl des Volumenersatzmittels einen Einfluss auf die Gerinnungsaktivität. Hydroxyethylstärke-haltige Präparate hemmen die Blutgerinnung stärker als kristalline Lösungen [9].

\section{Therapie der Koagulopathie}

Die ideale Therapie einer Dilutionskoagulopathie ist komplex und Gegenstand zahlreicher Diskussionen. Sie erfolgt durch die Gabe von gerinnungsaktiven Substanzen wie Faktorenkonzentraten (Fibrinogen, Prothrombinkomplexkonzentrat (PPSB), Faktor VIIa, Faktor XIII, FEIBA (Factor Eight Inhibitor Bypassing Activity)) oder Blutprodukten wie gefrorenem Frischplasma (fresh frozen plasma (FFP)) und Thrombozytenkonzentraten. Häufig ist die Kombination der genannten Substanzen indiziert.

\section{Rahmenbedingungen}

Auch in der postoperativen Phase steht die Optimierung der für die Gerinnung notwendigen Rahmenbedingungen an erster Stelle. Die zu optimierenden Parameter sind Temperatur, ionisiertes Kalzium, pH-Wert und der Hämatokritwert.

\section{Temperatur}

Die Enzyme des Gerinnungssystems folgen wie jeder biochemische Prozess der Reaktionsgeschwindigkeit-Temperatur-Regel. Pro 1 Grad Celsius Temperaturabfall sinkt die Aktivität der Gerinnungsenzyme um 4-10\%. Gleichzeitig nimmt die Aktivität der Thrombozyten bei Hypothermie signifikant ab [10]. Das Aufrechterhalten oder Wiederherstellen einer Normothermie ist daher von entscheidender Bedeutung.

\section{Kalzium}

Ionisiertes Kalzium (Faktor IV) ist als einziger Gerinnungsfaktor kein Protein. Es wird an verschiedenen Punkten der Hämostase als Kofaktor zwingend benötigt. Das in Erythroytenkonzentraten und FFP enthaltene Citrat bildet Komplexe mit Kalzium, sodass dieses nicht mehr für die Hämostase zur Verfügung steht. Die physiologische Konzentration des ionisierten Kalziums, das die biologisch aktive Form darstellt, beträgt 1,1-1,3 mmol/l. Im Blutungsfall sollte eine Konzentration von mehr als $0,9 \mathrm{mmol} / \mathrm{l}$ angestrebt werden [11]. 


\section{Säure-Basen-Status}

Die im Rahmen einer großen Volumenverschiebung häufig auftretende Gewebeminderperfusion geht in aller Regel mit einer Azidose einher. Die erhöhte $\mathrm{H}^{+}$-Aktivität führt zu einer weiteren Hemmung der Hämostase [12]. Ein pH-Wert unter 7,1 hat eine deutliche Verminderung der Gerinnungsaktivität zur Folge [11].

\section{Hämatokrit}

Die Adhäsion der Thrombozyten an die subendothelialen Strukturen ist abhängig von ihrer Präsenz am Rand des Blutgefäßes. Aufgrund komplexer strömungsmechanischer Vorgänge führt die Anwesenheit von Erythrozyten zu einer Verdrängung der wesentlich kleineren Thrombozyten aus der Gefäßmitte an den Rand [13], sodass die Wahrscheinlichkeit der Adhäsion steigt. Ein niedriger Hämatokrit hat eine verminderte Randverdrängung der Thrombozyten und somit eine reduzierte Adhäsionswahrscheinlichkeit zur Folge. Auch in der postoperativen Phase muss auf einen adäquaten ZielHämatokrit geachtet werden. In unterschiedlichen Empfehlungen findet sich bei größeren Blutverlusten sowie bei diffuser Blutung ein Ziel-Hämatokrit von $\geq 21 \%$ [14].

\section{Medikamentöse Therapie}

\section{Fibrinogen}

Fibrinogen ist als Substrat des Thrombins von entscheidender Bedeutung für die Stabilität des Gerinnsels. Die Substitution von Fibrinogen stellt in vielen Fällen einer erworbenen Koagulopathie die zentrale und wichtigste Intervention [15] dar und ist oftmals auch als alleinige Maßnahme zur Korrektur einer pathologischen Hämostase ausreichend. Die postinterventionelle Überwachung der Fibrinogenkonzentration ist im Blutungsfall daher unabdingbar. Trotz seiner frühen Ausschüttung als Protein der akuten Phase ist Fibrinogen im Rahmen verstärkter Blutungen der erste Gerinnungsfaktor, der eine kritische Konzentration erreicht [16]. Zur Therapie einer schweren Blutung sollten Fibrinogenspiegel von über 1,5 bzw. $2 \mathrm{~g} / \mathrm{l}$ angestrebt werden [17]. Diese Werte können mit der Transfusion von FFP alleine kaum erreicht werden [18]. Daher sollte in Blutungssituationen auf Fibrinogenkonzentrat zurückgegriffen werden, um adäquate Plasmaspiegel zu erreichen. Die zu substituierende Fibrinogenmenge in Gramm wird berechnet, indem der gewünschte Anstieg in g/l mit dem Plasmavolumen in Liter (beträgt ca. $40 \mathrm{ml}$ pro Kilogramm Körpergewicht (kgKG)) multipliziert wird.

\section{FFP}

FFP enthält alle Komponenten des plasmatischen Gerinnungssystems und kann daher zum Ersatz verbrauchter Gerinnungsfaktoren verwendet werden. Die Indikation zum Ein- satz von FFP ist nach den Querschnittsleitlinien zur Therapie mit Blutkomponenten und Plasmaderivaten der Bundesärztekammer bei einem schweren akuten Blutverlust und einem Quick-Wert $<50 \%$ gegeben [19]. Als Dosierung werden 15-20 $\mathrm{ml} / \mathrm{kgKG}$ FFP angegeben; dies ist jedoch ohne hinreichende Evidenz, und es können auch deutlich größere Volumina benötigt werden. Zur laborgesteuerten Gerinnungsoptimierung werden $1 \mathrm{ml} \mathrm{FFP/kgKG} \mathrm{für} \mathrm{eine} \mathrm{Erhöhung} \mathrm{des} \mathrm{Quick-Werts}$ um $1 \%$ empfohlen. Nachteilig sind die schlechte logistische Verfügbarkeit (Lagerung in der Blutbank, Zeitverlust durch Auftauen und Transport), die niedrige Fibrinogenkonzentration sowie die hohen benötigten Volumina. Zudem ist die Gabe von FFP risikobehaftet. Zu den Risiken zählen unter anderem das transfusionsassoziierte Lungenversagen [20], Transfusionsreaktionen, mögliche AB0-Inkompatibilität [21] sowie ein erhöhtes postoperatives Infektionsrisiko [22].

\section{Thrombozyten}

Die Querschnittsleitlinien der Bundesärztekammer empfehlen, bei massiven und bedrohlichen sowie bei transfusionspflichtigen Blutungen eine Thrombozytenzahl $\geq 10^{5} / \mu \mathrm{l}$ anzustreben [19]. In Situationen mit geringem Blutverlust scheinen $5 \times 10^{4} / \mu \mathrm{l}$ ausreichend zu sein. Diese rein quantitativen Werte berücksichtigen jedoch nicht die Thrombozytenfunktion, die durch angeborene (z.B. vWS), medikamentöse, äußere (siehe «Rahmenbedingungen») oder mechanische (extrakorporale Zirkulation) Einflüsse beeinträchtigt sein kann. Eine reduzierte Thrombozytenfunktion kann durch die Gabe von Desmopressin $(0,3 \mu \mathrm{g} /$ $\mathrm{kgKG}$ ) verbessert werden. Des Weiteren kann die Substitution von Fibrinogen eine reduzierte Thrombozytenaktivität bis zu einem gewissen Grad kompensieren [23].

\section{Faktor XIII}

Der fibrinstabilisierende Faktor XIII fördert die Fibrinquervernetzung und reduziert die Fibrinolyse. Eine erniedrigte Konzentration von Faktor XIII ist mit einer erhöhten postoperativen Blutungsneigung vergesellschaftet [24]. Für einen effektiven Einsatz von Faktor XIII muss ausreichend Fibrinogen vorhanden sein. In der postoperativen Phase sollte eine Faktor-XIII-Aktivität von $\geq 60 \%$ angestrebt werden [25].

\section{Prothrombinkomplexkonzentrat}

PPSB enthält die Vitamin-K-abhängigen Gerinnungsfaktoren II, VII, IX und X sowie die antikoagulatorisch wirksamen Faktoren Protein S, Protein C und Antithrombin. Den meisten Präparaten ist Heparin zugefügt, das zumindest in vitro auch eine Wirkung auf die Blutgerinnung hat [26]. Das ursprüngliche Einsatzgebiet von PPSB ist die Reversierung 
einer bestehenden Antikoagulation mit Vitamin-K-Antagonisten, allerdings beschreiben retrospektive Studien sowie experimentelle Arbeiten am Tiermodell einen erfolgreichen Einsatz im perioperativen Umfeld [27, 28]. Eine Einheit PPSB/kgKG erhöht den Quick-Wert um 1\%.

\section{Rekombinanter aktivierter Faktor VII}

Rekombinanter aktivierter Faktor VII (rFVIIa) ist in Deutschland nur zur Therapie bestimmter angeborener oder erworbener Hämophilien zugelassen. In den letzten Jahren wurde der erfolgreiche Off-label-Einsatz auch bei lebensbedrohlichen Blutungen beschrieben [29]. Es kommt unabhängig vom Gewebefaktor zu einer Thrombingenerierung. Ein Einsatz von rFVIIa ist im Rahmen einer Koagulopathie nur dann sinnvoll, wenn gleichzeitig ausreichend Thrombinsubstrat, also Fibrinogen, zur Verfügung steht. Wenn sämtliche anderen prokoagulatorischen Therapien gescheitert sind, kann ein Therapieversuch mit einer Bolusgabe von 80-120 $\mu \mathrm{g} /$ $\mathrm{kgKG}$ nach sorgfältiger Nutzen-Risiko-Abwägung bei persistierender postoperativer Blutung durchgeführt werden.

\section{Antifibrinolytika}

Bei Koagulopathien mit großem Volumenumsatz besteht das Risiko einer Hyperfibrinolyse. Goldstandard zur zügigen Detektion ist die Thromboelastographie/Thromboelastometrie. Nachdem Aprotinin 2008 vom Markt genommen wurde, steht in Deutschland als Antifibrinolytikum nur noch Tranexamsäure zur Verfügung. Tranexamsäure blockiert die Lysinbindungsstelle des Plasminogens. Unter anderem durch die CRASH-II-Studie [30] ist Tranexamsäure in den letzten Jahren als kostengünstiges, effektives und nebenwirkungsarmes Medikament in den Fokus geraten. Als Dosis hat sich bisher ein Bolus von 1-2 g gefolgt von $1-5 \mathrm{mg} / \mathrm{kg} / \mathrm{h}$ etabliert [31]; die Dosierung ist jedoch ohne hinreichende Evidenz. Die Indikation zur postoperativen Gabe von Tranexamsäure bei Blutungskomplikationen ist bis heute nicht eindeutig bewiesen und kann deshalb zum jetzigen Zeitpunkt nur nach sorgfältiger Indikationsstellung empfohlen werden.

\section{Diagnostik der Blutgerinnung}

Die Blutgerinnung ist ein In-vivo-Ereignis, das von verschiedensten Faktoren abhängt und ein komplexes Zusammenspiel von pro- und antikoagulatorischen Einflüssen darstellt. Die unterschiedlichen teilnehmenden Bestandteile sind zellulär, molekular, atomar, organisch und anorganisch. Das Monitoring der Gerinnung kann daher niemals den gesamten Gerinnungsprozess abbilden, sondern immer nur einzelne Teile erfassen.

\section{Klassische Gerinnungstests}

Als klassische Gerinnungstests werden Prothrombinzeit (Quick, International Normalized Ratio (INR)), aktivierte partielle Thromboplastinzeit (aPTT) und Thrombozytenzahl verstanden. Die Bestimmung von Quick und aPTT wurden entwickelt, um die Wirkung von Vitamin-K-Antagonisten bzw. Heparin zu überwachen. Diesen Tests ist gemeinsam, dass sie im Labor unter standardisierten Bedingungen (37 Grad Celsius, $\mathrm{Ca}^{2+}$-Überschuss, gepufferte Lösung) durchgeführt werden. Sie sind also von den In-vivo-Bedingungen meist völlig entkoppelt. Ein azidotischer, hypothermer Patient mit $\mathrm{Ca}^{2+}$-Mangel wird im Gerinnungslabor daher zunächst keine pathologischen Werte aufweisen. Eine weitere Limitation ist, dass sie nur die initiale Formierung eines Clots abbilden, während eine Aussage über die Qualität des Clots, seine Lyse sowie den Einfluss der korpuskulären Bestandteile der Gerinnung nicht getroffen werden kann. Eine Vorhersage über die Blutungswahrscheinlichkeit bei invasiven Prozeduren ist mit ihrer Hilfe nicht ausreichend möglich [32]. Des Weiteren sind die klassischen Gerinnungstests nicht bettseitig verfügbar, sodass auch das Ergebnis nicht zeitnah verfügbar ist [33].

\section{Point-of-Care-Diagnostik}

Die Point-of-Care(POC)-Diagnostik zeichnet sich dadurch aus, dass die Untersuchung außerhalb des Zentrallabors («bettseitig») durchgeführt werden kann. Beispiele für POC-Geräte im OP-Alltag sind die Activated Clotting Time (ACT) oder die Blutgasanalyse (BGA). Das wohl wichtigste POC-Gerät für die Gerinnungsdiagnostik ist die viskoelastische Beurteilung des Clots mittels Thromboelastographie bzw. Thromboelastometrie (ROTEM ${ }^{\circledR}$; Tem International GmbH, München, Deutschland). Hierbei ist es möglich, die Dynamik von Clotentstehung, -stärke und -lyse zu erfassen. Dadurch können verschiedene Ursachen einer Koagulopathie wie Faktorenmangel, Thrombozytenmangel und -funktionsstörung, Fibrinogenmangel, Hyperfibrinolyse oder eine Heparinwirkung in Echtzeit erfasst werden. Erste hinweisende Ergebnisse sind bereits wenige Minuten nach Testbeginn verfügbar [34]. Eine Gerinnungstherapie, die durch viskoelastische Tests gesteuert wird, ist daher in der Lage, den Verbrauch an Blutprodukten sowie die Mortalität zu senken $[28,35]$. Ein Diagnostikalgorithmus wird in Abbildung 1 gezeigt. Eine Limitation der viskoelastischen Tests ist jedoch, dass sie die Wirkung von plättchenhemmenden Medikamenten nicht erfassen können. Eine Ausnahme bildet hier der TEG ${ }^{\circledR}$ 5000 Platelet Mapping ${ }^{\mathrm{TM}}$ Assay.

\section{Antikoagulation}

Die Zahl der Verschreibungen von Antikoagulanzien steigt [5]. Patienten, die sich unter Antikoagulation einem 


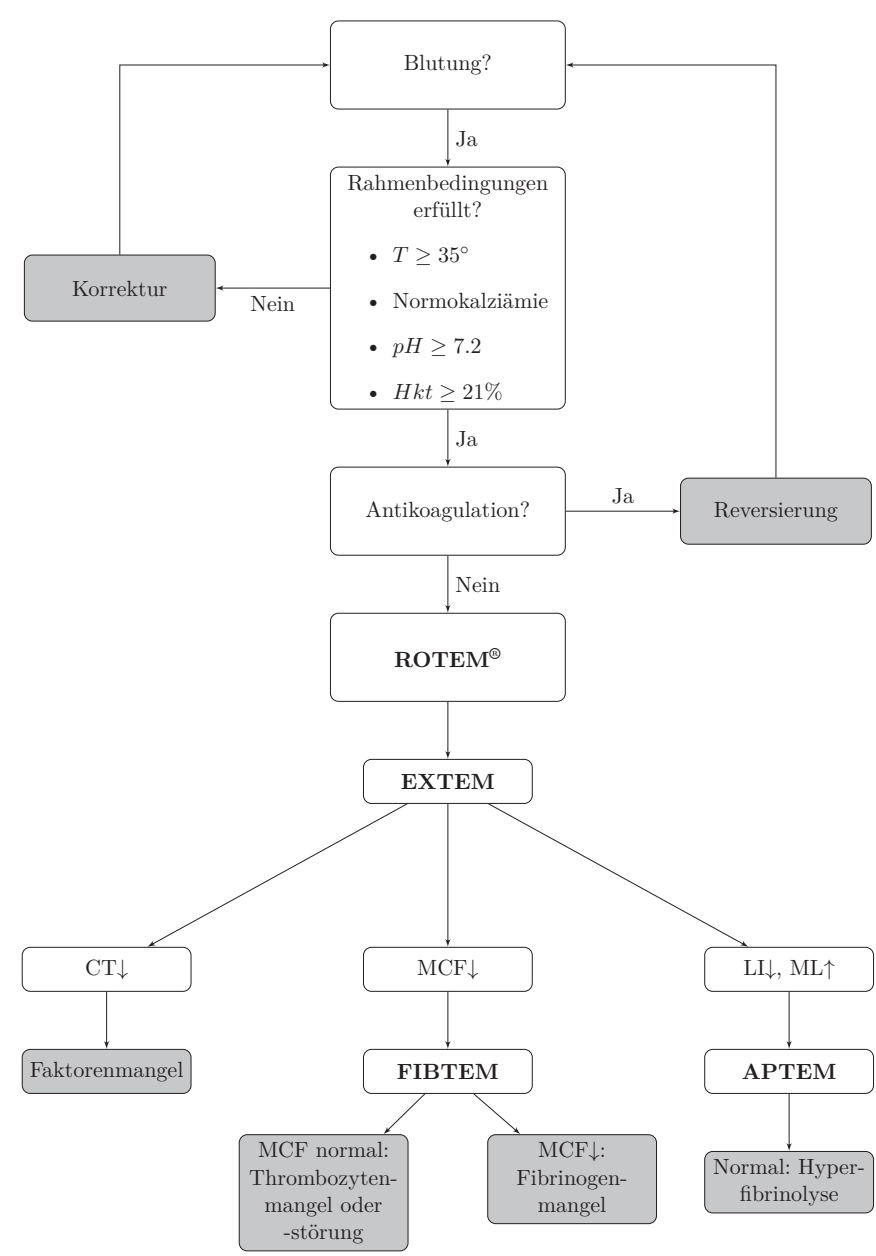

Abb. 1. Thromboelastometrie-basierter Algorithmus zur Diagnostik einer postoperativen Blutung. Abkürzungen: $\mathrm{CT}=$ Clotting Time/Gerinnungszeit, $\mathrm{MCF}=$ Maximum Clot Firmness, $\mathrm{LI}=$ Lysis Index, ML $=\mathrm{Ma}-$ ximum Lysis.

operativen Eingriff unterziehen müssen, stellen im perioperativen Management eine besondere Herausforderung dar. Einerseits soll das Risiko von Blutungen minimiert werden, andererseits stellt die Zeitdauer ohne Antikoagulation ein Risiko für thromboembolische Ereignisse dar. Die Angriffspunkte der Medikamente in der Gerinnungskaskade sind vielseitig und reichen von der Plättchenaggregationshemmung (Acetylsalicylsäure (ASS), Clopidogrel) über die Synthesehemmung Vitamin-K-abhängiger Faktoren (Phenprocoumon) bis zur Hemmung von Faktoren der Gerinnungskaskade selbst (unfraktionierte und niedermolekulare Heparine). Zusätzlich sind in den letzten Jahren zahlreiche neue orale Antikoagulanzien sowie neue orale Thrombozytenaggregationshemmer auf den Markt gekommen, die sich von den bekannten Medikamenten in Pharmakodynamik und Pharmakokinetik deutlich unterscheiden. Das Management von Patienten unter Antikoagulation in der perioperativen Phase ist abhängig von dem Blutungsrisiko des geplanten Eingriffs sowie dem individuellen Risiko für thromboembolische Ereignisse. Handlungsempfehlungen für die Umstellung der An- tikoagulation sowie ein Bridging sind an anderer Stelle beschrieben [36].

\section{Postoperative Fortsetzung einer bestehenden Antikoagulation}

Vitamin-K-Antagonisten werden in der Regel zur Prävention von thromboembolischen Ereignissen bei persistierendem Vorhofflimmern, nach Implantation von mechanischen Herzklappenprothesen sowie zur Rezidivprophylaxe nach Venenthrombosen verwendet. Ihr Wirkmechanismus beruht auf der Synthesehemmung der Vitamin-K-abhängigen Gerinnungsfaktoren (II, VII, IX und X). Ein therapeutischer antikoagulativer Effekt wird daher erst nach 5-7 Tagen erreicht. Die Fortführung einer Therapie kann noch am Abend oder am Folgetag des operativen Eingriffs erfolgen. Bei Eingriffen mit hohem Blutungsrisiko sollte die Therapie erst nach 1-2 Tagen fortgeführt werden [36].

\section{Thrombozytenaggregationshemmer}

Thrombozytenaggregationshemmer werden meistens zur Prävention einer Stent- oder Gefäßthrombose nach Koronarintervention verschrieben. Nach einer Implantation von Koronarstents ist die doppelte Antiplättchentherapie (DAPT) mit ASS sowie einem P2Y12-Rezeptor-Blocker als Bestandteil mehrerer Leitlinien zwingend erforderlich [37]. Die ASSTherapie wird normalerweise lebenslang fortgeführt und sollte auch perioperativ nur in gut begründeten Ausnahmefällen unterbrochen werden. Die «klassische» DAPT erfolgt mit ASS und Clopidogrel. Die postoperative Fortführung der Einnahme von Thrombozytenaggregationshemmern sollte abhängig vom Eingriff und der Blutungssituation in Rücksprache mit dem Operateur so schnell wie möglich erfolgen. In den letzten Jahren sind vermehrt neue Thrombozytenaggregationshemmer auf den Markt gekommen, die sich in ihren pharmakologischen Eigenschaften von Clopidogrel und ASS unterscheiden. Als wichtigste Vertreter werden hier Prasugrel (Efient ${ }^{\circledR}$; Lilly Deutschland GmbH, Bad Homburg, Deutschland) und Ticagrelor (Brilique $^{\circledR}$; AstraZeneca GmbH, Wedel, Deutschland) beschrieben. Prasugrel ist wie Clopidogrel ein irreversibler P2Y12-Rezeptor-Blocker, unterscheidet sich von diesem jedoch unter anderem durch einen schnelleren Wirkungseintritt sowie eine stärkere Wirkung [38, 39]. Ticagrelor hingegen ist ein reversibler direkter ADP-Rezeptor-Inhibitor.

\section{Neue orale Antikoagulanzien}

Die sogenannten neuen oralen Antikoagulanzien beinhalten den direkten Thrombininhibitor Dabigatran (Pradaxa ${ }^{\circledR}$; Boehringer Ingelheim Pharma GmbH, Ingelheim am Rhein, 
Deutschland) sowie den Faktor-Xa-Inhibitor Rivaroxaban (Xarelto ${ }^{\circledR}$; Bayer AG, Leverkusen, Deutschland). Die neuen oralen Antikoagulanzien zeichnen sich durch eine hohe orale Bioverfügbarkeit und einen schnellen Wirkungseintritt aus [40]. Bei der Gabe von oralen Substanzen muss bedacht werden, dass die Resorption durch die reduzierte Darmmotilität nach viszeralchirurgischen Eingriffen reduziert sein kann. Die klassische Gerinnungsdiagnostik kann Veränderungen der Antikoagulation durch diese Substanzen nicht hinreichend erfassen. Die Überwachung von Dabigatran ist durch Messung der nicht sehr verbreiteten Ecarin Clotting Time (ECT) möglich; die Rivaroxaban-Aktivität lässt sich durch den chromogenen Anti-Xa-Test messen. Eine Übersicht über die Wirkung der Antikoagulanzien auf die Gerinnungsdiagnostik gibt Tabelle 1. Der postoperative Wiederbeginn einer Therapie mit Dabigatran und Rivaroxaban erfolgt abhängig vom thromboembolischen und chirurgischen Risikoprofil des Patienten [41].
Tab. 1. Beeinflussung der Gerinnungsdiagnostik durch die neuen Antikoagulanzien (modifiziert nach [42])

\begin{tabular}{lll}
\hline Test & Dabigatran & Rivaroxaban \\
\hline Quick & $\downarrow$ & $\downarrow(\downarrow)$ \\
aPTT & $\uparrow \uparrow$ & $\uparrow$ \\
Thrombinzeit & $\uparrow \uparrow \uparrow$ & $\varnothing$ \\
ACT & $\uparrow$ & $\uparrow$ \\
ECT & $\uparrow \uparrow$ & $\varnothing$ \\
Chromogener Anti-Xa-Assay & $\varnothing$ & $\uparrow \uparrow$ \\
Chromogener Anti-IIa-Assay & $\uparrow \uparrow$ & $\varnothing$ \\
\hline
\end{tabular}

aPTT = Aktivierte partielle Thromboplastinzeit; ACT = aktivierte Gerinnungszeit; ECT = Ecarin-Gerinnungszeit.

\section{Disclosure Statement}

Keine Interessenkonflikte.

\section{Literatur}

1 Turan A, Yang D, Bonilla A, Shiba A, Sessler DI, Saager L, Kurz A: Morbidity and mortality after massive transfusion in patients undergoing noncardiac surgery. Can J Anaesth 2013;60:761-770.

2 Unalp HR, Kamer E, Kar H, Bal A, Peskersoy M, Ali Onal M: Urgent abdominal re-explorations. World J Emerg Surg 2006;1:10.

$\$ 3$ Grant PF: Hormonal regulation of the acute haemostatic response to stress. Blood Coagul Fibrinolysis 1990;1:299-306

4 Haas S, Encke A, Krauspe R, Waydhas C: Thromboseprophylaxe in der Chirurgie. Chirurg 2011;82: 1085-1090.

$\checkmark 5$ Wysowski DK, Nourjah P, Swartz L: Bleeding complications with warfarin use: a prevalent adverse effect resulting in regulatory action. Arch Intern Med 2007;167:1414-1419.

6 McLoughlin TM, Fontana JL, Alving B, Mongan PD, Bünger R: Profound normovolemic hemodilution: hemostatic effects in patients and in a porcine model. Anesth Analg 1996;83:459-465.

7 Vischer UM, Wollheim CB: Epinephrine induces von Willebrand factor release from cultured en dothelial cells: involvement of cyclic AMP-dependent signalling in exocytosis. Thromb Haemost 1997; 77:1182-1188.

8 Jesty J, Beltrami E: Positive feedbacks of coagulation: their role in threshold regulation. Arterioscler Thromb Vasc Biol 2005;25:2463-2469.

$\checkmark$ Brummel-Ziedins K, Whelihan MF, Ziedins EG, Mann KG: The resuscitative fluid you choose may potentiate bleeding. J Trauma 2006;61:1350-1358.

10 Watts DD, Trask A, Soeken K, Perdue P, Dols S, Kaufmann C: Hypothermic coagulopathy in trauma: effect of varying levels of hypothermia on enzyme speed, platelet function, and fibrinolytic activity. J Trauma 1998;44:846-854

11 Lier H, Krep H, Schroeder S, Stuber F: Preconditions of hemostasis in trauma: a review. The influence of acidosis, hypocalcemia, anemia, and hypothermia on functional hemostasis in trauma. J Trauma 2008;65:951-960.
12 Hoyt DB, Dutton RP, Hauser CJ, Hess JR, Holcomb JB, Kluger Y, Mackway-Jones K, Parr MJ, Rizoli SB, Yukioka T, Bouillon B: Management of coagulopathy in the patients with multiple injuries: results from an international survey of clinical practice. J Trauma 2008;65:755-764; discussion 764-765.

13 Goldsmith HL: The Microcirculatory Society Eugene M. Landis Award lecture. The microrheology of human blood. Microvasc Res 1986;31:121-142.

14 Theusinger OM, Spahn DR, Ganter MT: Transfusion in trauma: why and how should we change our current practice? Curr Opin Anaesthesiol 2009;22: 305-312.

15 Levy JH, Szlam F, Tanaka KA, Sniecienski RM: Fibrinogen and hemostasis: a primary hemostatic target for the management of acquired bleeding. Anesth Analg 2012;114:261-274.

16 Hiippala ST, Myllylä GJ, Vahtera EM: Hemostatic factors and replacement of major blood loss with plasma-poor red cell concentrates. Anesth Analg 1995;81:360-365.

17 Spahn DR, Bouillon B, Cerny V, Coats TJ, Duranteau J, Fernandez-Mondejar E, Filipescu D, Hunt BJ, Komadina R, Nardi G, Neugebauer E, Ozier Y, Riddez L, Schultz A, Vincent J-L, Rossaint R: Management of bleeding and coagulopathy following major trauma: an updated European guideline. Crit Care 2013;17:R76.

18 Chowdary P, Saayman AG, Paulus U, Findlay GP, Collins PW: Efficacy of standard dose and $30 \mathrm{ml} / \mathrm{kg}$ fresh frozen plasma in correcting laboratory parameters of haemostasis in critically ill patients. $\mathrm{Br}$ J Haematol 2004;125:69-73.

19 www.bundesaerztekammer.de/downloads/Querschnittsleitlinie_Gesamtdokument deutsch_07032011. pdf. 2011.

20 Toy P, Lowell C: TRALI - definition, mechanisms, incidence and clinical relevance. Best Pract Res Clin Anaesthesiol 2007:21:183-193.
1 Shanwell A, Andersson TM, Rostgaard K, Edgren G, Hjalgrim H, Norda R, Melbye M, Nyrén O, Reilly M: Post-transfusion mortality among recipients of ABO-compatible but non-identical plasma. Vox Sang 2009;96:316-323.

22 Sarani B, Dunkman WJ, Dean L, Sonnad S, Rohrbach JI, Gracias VH: Transfusion of fresh frozen plasma in critically ill surgical patients is associated with an increased risk of infection. Crit Care Med 2008;36:1114-1118.

23 Velik-Salchner C, Haas T, Innerhofer P, Streif W, Nussbaumer W, Klingler A, Klima G, Martinowitz U, Fries D: The effect of fibrinogen concentrate on thrombocytopenia. J Thromb Haemost 2007;5:10191025.

24 Korte WC, Szadkowski C, Gähler A, Gabi K, Kownacki E, Eder M, Degiacomi P, Zoller N, Devay J, Lange J, Schnider T: Factor XIII substitution in surgical cancer patients at high risk for intraoperative bleeding. Anesthesiology 2009;110:239-245.

25 Korte W: F. XIII in perioperative coagulation management. Best Pract Res Clin Anaesthesiol 2010; 24:85-93.

26 Scharbert G, Thaler U, Weilnböck C, Wetzel L, Kozek-Langenecker S: Heparin-induced effects of prothrombin complex concentrates in thromboelastometry. Wien Klin Wochenschr 201;124:320-325.

27 Dickneite G, Pragst I: Prothrombin complex concentrate vs fresh frozen plasma for reversal of dilutional coagulopathy in a porcine trauma model. $\mathrm{Br}$ J Anaesth 2009;102:345-354.

28 Schöchl H, Nienaber U, Maegele M, Hochleitner G, Primavesi F, Steitz B, Arndt C, Hanke A, Voelckel W, Solomon C: Transfusion in trauma: thromboelastometry-guided coagulation factor concentrate-based therapy versus standard fresh frozen plasma-based therapy. Crit Care 2011;15:R83.

29 Grottke O, Henzler D, Rossaint R: Activated recombinant factor VII (rFVIIa). Best Pract Res Clin Anaesthesiol 2010;24:95-106. 
-30 CRASH-2 trial collaborators; Shakur H, Roberts I, Bautista R, et al.: Effects of tranexamic acid on death, vascular occlusive events, and blood transfusion in trauma patients with significant haemorrhage (CRASH-2): a randomised, placebo-controlled trial. Lancet 2010;376:23-32.

-31 Cap AP, Baer DG, Orman JA, Aden J, Ryan K, Blackbourne LH: Tranexamic acid for trauma patients: a critical review of the literature. J Trauma 2011;71(suppl 1):S9-14.

-32 Segal JB, Dzik WH; Transfusion Medicine/Hemostasis Clinical Trials Network: Paucity of studies to support that abnormal coagulation test results predict bleeding in the setting of invasive procedures: an evidence-based review. Transfusion 2005;45:1413-1425.

-33 Toulon P, Ozier Y, Ankri A, Fléron M-H, Leroux G, Samama CM: Point-of-care versus central laboratory coagulation testing during haemorrhagic surgery. A multicenter study. Thromb Haemost 2009;101:394-401.

34 Pezold M, Moore EE, Wohlauer M, Sauaia A, Gonzalez E, Banerjee A, Silliman CC: Viscoelastic clot strength predicts coagulation-related mortality within 15 minutes. Surgery 2012;151:48-54.
35 Weber CF, Görlinger K, Meininger D, Herrmann E, Bingold T, Moritz A, Cohn LH, Zacharowski K: Point-of-care testing: a prospective, randomized clinical trial of efficacy in coagulopathic cardiac surgery patients. Anesthesiology 2012;117:531-547.

36 Douketis JD, Spyropoulos AC, Spencer FA, Mayr M, Jaffer AK, Eckman MH, Dunn AS, Kunz R; American College of Chest Physicians: Perioperative management of antithrombotic therapy: Antithrombotic Therapy and Prevention of Thrombosis, 9th ed: American College of Chest Physicians Evidence-Based Clinical Practice Guidelines. Chest 2012;141(suppl 2):e326S-e350S.

37 Task Force on Myocardial Revascularization of the European Society of Cardiology (ESC) and the European Association for Cardio-Thoracic Surgery (EACTS); European Association for Percutaneous Cardiovascular Interventions (EAPCI); Kolh P, Wijns W, Danchin N, et al.: Guidelines on myocardial revascularization. Eur J Cardiothorac Surg 2010;38(suppl):S1-S52.
38 Jernberg T, Payne CD, Winters KJ, Darstein C, Brandt JT, Jakubowski JA, Naganuma H, Siegbahn A, Wallentin L: Prasugrel achieves greater inhibition of platelet aggregation and a lower rate of non-responders compared with clopidogrel in aspirin-treated patients with stable coronary artery disease. Eur Heart J 2006;27:1166-1173.

39 Jakubowski JA, Payne CD, Brandt JT, Weerakkody GJ, Farid NA, Small DS, Naganuma H, Li GY, Winters KJ: The platelet inhibitory effects and pharmacokinetics of prasugrel after administration of loading and maintenance doses in healthy subjects. J Cardiovasc Pharmacol 2006;47:377-384.

40 Eriksson BI, Quinlan DJ, Weitz JI: Comparative pharmacodynamics and pharmacokinetics of oral direct thrombin and factor Xa inhibitors in development. Clin Pharmacokinet 2009;48:1-22.

41 Kozek-Langenecker SA, Afshari A, Albaladejo P, et al.: Management of severe perioperative bleeding: guidelines from the European Society of Anaesthesiology. Eur J Anaesthesiol 2013;30:270-382.

42 Mani H, Kasper A, Lindhoff-Last E: Measuring the anticoagulant effects of target specific oral anticoagulants - reasons, methods and current limitations. J Thromb Thrombolysis 2013;36:187-194. 